\title{
Cross-Dehydrogenative Coupling Reaction and Arylation of Quinoxalin-2(1H)-ones under lodide/Peroxide Conditions
}

\author{
Yujuan $\mathbf{W u}^{\mathrm{a}}$ \\ Xianglong $\mathrm{Chu}^{\mathrm{a}}$ \\ Di Yang ${ }^{\mathrm{a}}$ \\ Chen Ma*a (i) \\ Caixia Xie ${ }^{\mathrm{b}}$ \\ ${ }^{a}$ Institute of Organic Chemistry, School of Chemistry and \\ Chemical Engineering, Shandong University, Jinan 250100, \\ P. R. of China \\ chenma@sdu.edu.cn \\ b School of Chemistry and Chemical Engineering, Shandong \\ University of Technology, Zibo 255049, P. R. of China
}
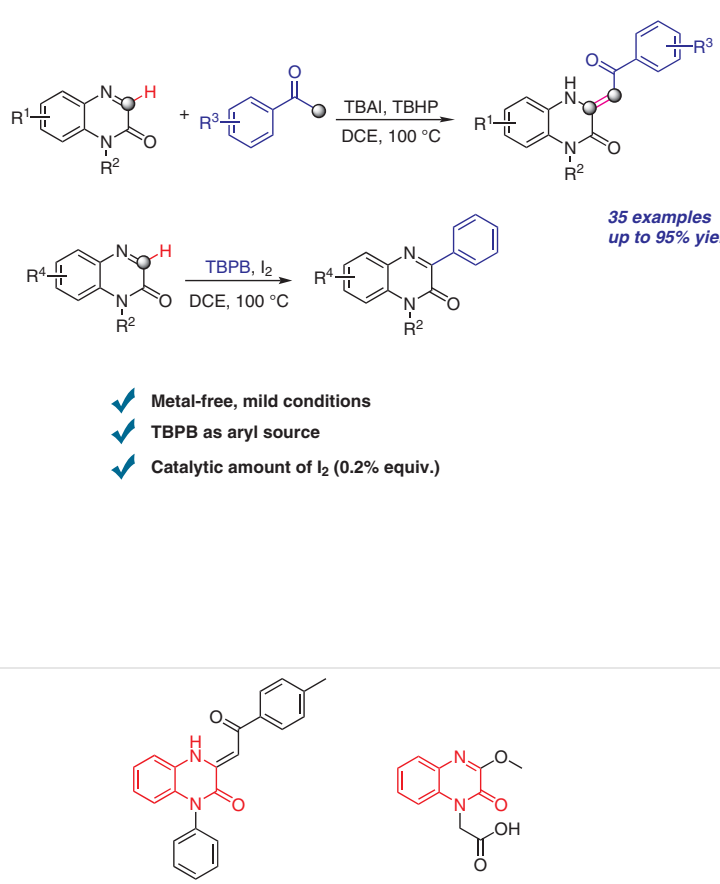
35 examples
up to $95 \%$ yield

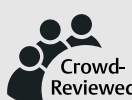

Published online: 23.04 .2021

DOI: 10.1055/a-1489-8711; Art ID: so-2021-d0013-I

License terms: (cc)

(c) 2021. The Author(s). This is an open access article published by Thieme under the terms of the Creative Commons Attribution-NonDerivative-NonCommercial-License, permitting copying and reproduction so long as the original work is given appropriate credit. Contents may not be used for commercial purposes or adapted, remixed, transformed or built upon. (https://creativecommons.org/licenses/by-nc-nd/4.0/)

Abstract A simple method has been developed for the synthesis of 3-(2-oxo-2-phenylethylidene)-3,4-dihydroquinoxalin-2(1H)-one and 3-aryl-quinoxalin-2(1H)-one derivatives through $\mathrm{C}-\mathrm{H}$ activation of quinoxalin-2(1H)-ones by peroxides and iodide. In this protocol, the peroxide (TBPB) serves as both the radical initiator and aryl source, realizing arylation of quinoxalin-2(1H)-one in a one-step reaction. The methodology has the advantages of being a metal-free strategy and having broad functional group tolerance.

Key words $\mathrm{C}-\mathrm{H}$ activation, iodide/peroxide system, metal-free, quinoxalin-2(1H)-one, radical process

Quinoxalin-2(1H)-one scaffolds exist in many natural products. Due to the significant biological activities and pharmaceutical properties of this structure, such as antitumor, ${ }^{1}$ ALR2 inhibition, ${ }^{2}$ antibiotic, ${ }^{3}$ analgesic, ${ }^{4}$ antimicrobial, ${ }^{5}$ and aldose reductase inhibition activities (Figure 1 ), ${ }^{6}$ a range of 3-functionalized quinoxalin-2(1H)-ones has been synthesized, with approaches including arylation, ${ }^{7}$ alkylation, ${ }^{8}$ etherification, ${ }^{9}$ amidation, ${ }^{10}$ amination, ${ }^{11}$ cyanation, ${ }^{12}$ phosphonation, ${ }^{13}$ and trifluoromethylation (Scheme 1). ${ }^{14}$

Activation of $\mathrm{C}-\mathrm{H}$ bonds has recently emerged as a powerful method for the construction of C-C bonds. ${ }^{15,16}$ Furthermore, radical addition is a powerful method to form $\mathrm{C}-\mathrm{C}$ bonds, ${ }^{17}$ with the iodide/TBHP system being considered as a dominant protocol in the radical field to achieve $\mathrm{C}-\mathrm{H}$ bond activation and construction of heterocyclic rings. ${ }^{18,19}$

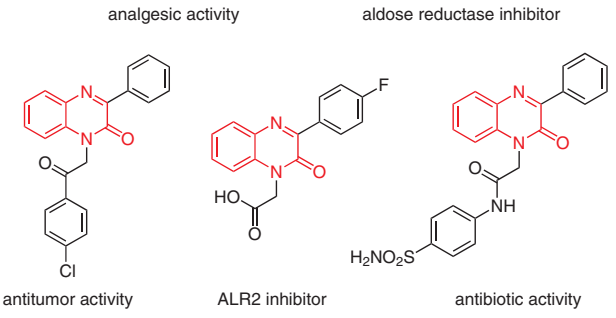

Figure 1 Pharmaceutically active quinoxalin-2(1H)-one derivatives

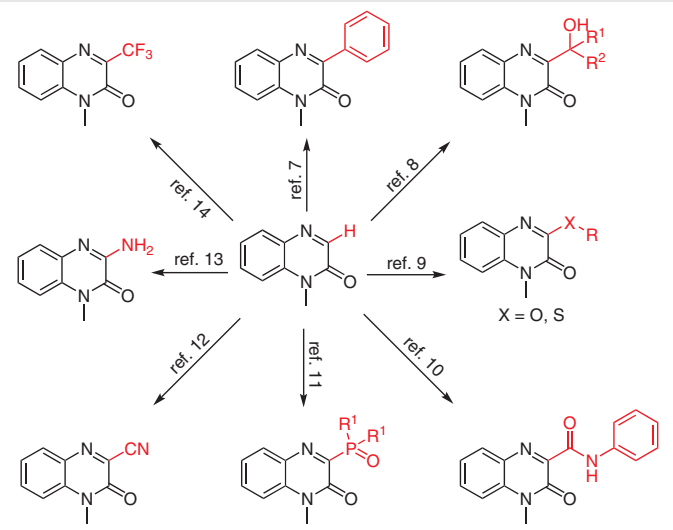

Scheme 1 Synthesis of 3-functionalized quinoxalin-2(1H)-one derivatives 
Typical approaches for synthesizing 3-(2-oxo-2-phenylethylidene)-3,4-dihydroquinoxalin-2(1H)-ones involve reaction of aroyl- and hetaroylpyruvic acid or ester derivatives with $\mathrm{N}$-phenyl-o-phenylenediamine (Scheme 2). ${ }^{20,21}$

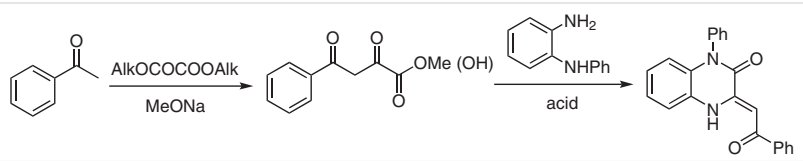

Scheme 2 Synthesis of 3-acetylquinoxalin-2(1H)-one derivatives

As for the synthesis of diverse 3-aryl-quinoxalin-2(1H)ones, there were two main approaches. One strategy to construct the heterocyclic ring involves two-step acylation of benzene-1,2-diamines with arylglyoxylic acids, followed by subsequent cyclization (Scheme 3 , method 1 ). ${ }^{7 a}$ Other methods involve direct functionalization. Paul reported the novel Pd(TFA) ${ }_{2}$-catalyzed direct dehydrogenative cross-coupling of quinoxalin-2-ones with arenes for the construction of diverse 3-aryl quinoxalin-2-ones (Scheme 3, method 2 ). ${ }^{7 b}$ Ramesh reported an oxidative cross-coupling of arylboronic acids with quinoxalin-2-ones using the readily available oxidant $\mathrm{Mn}(\mathrm{III})$ acetate dihydrate (Scheme 3, method 3). ${ }^{7 \mathrm{C}}$ Yin' s group used diaryliodonium tetrafluoroborates at room temperature, with arylhydrazines as the arylating agent (Scheme 3 , method 4). ${ }^{7 \mathrm{~d}}$ Lee and co-workers reported iodosobenzene-promoted direct arylation with arylhydrazines as radical precursors (Scheme 3 , method 5). ${ }^{7 e}$ However, drawbacks such as the requirement for prefunctionalized substrates, multistep protocols, low atom economy, use of transition-metal catalysts and strong base in these protocols have limited their general application and development. Metal-free systems have replaced tradition metal systems, and iodide or hypervalent iodine possess advantages such as ease of handling, strong electrophilicity, commercial availability, and low toxicity. ${ }^{22}$

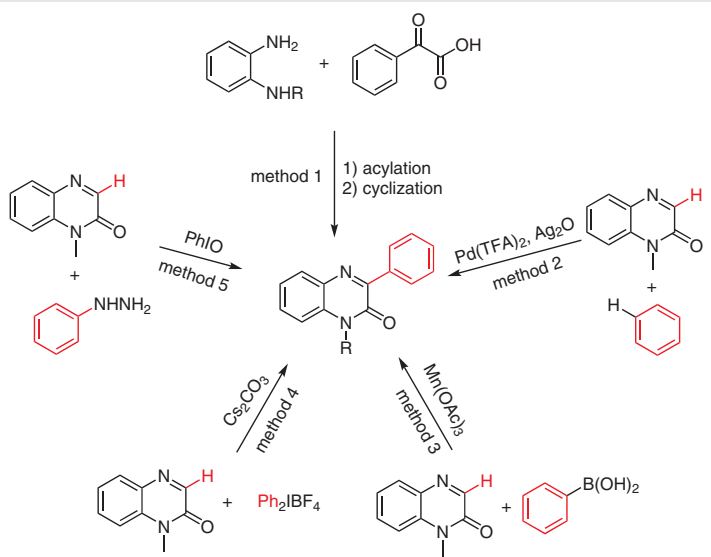

Scheme 3 Synthetic approaches to 3-arylquinoxalin-2(1H)-ones
The $\mathrm{I}_{2} / \mathrm{TBHP}$ system is a central reagent combination in the radical field. ${ }^{23,24}$ It has been shown that tert-butyl peroxybenzoate (TBPB) is an efficient and highly chemoselective benzoylating reagent. ${ }^{25,26}$ In this area, we first disclosed that TBPB could translate into aryl radicals, triggering subsequent reactions, providing a novel method for the introduction of an aryl group.

Herein, we disclose a simple method to synthesize 3functionalized quinoxalin-2(1H)-ones. In the first part, we present a $n-\mathrm{Bu}_{4} \mathrm{NI}$-catalyzed radical oxidative coupling of acetophenone and quinoxalin-2(1H)-ones using TBHP as oxidant to access 3-(2-oxo-2-phenylethylidene)-3,4-dihydroquinoxalin-2 $(1 H)$-ones. In the second part, the direct arylation of quinoxalin-2(1H)-ones is disclosed. Therein, TBPB is used both as reagent to generate aryl radical and as free radical initiator, while $\mathrm{I}_{2}$ is used as catalyst (Scheme 4).

(a)

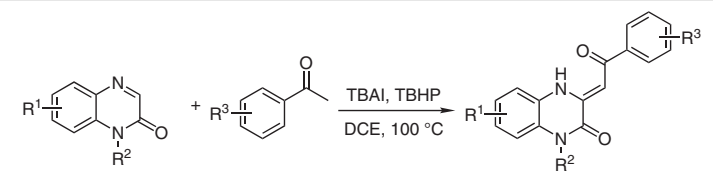

(b)

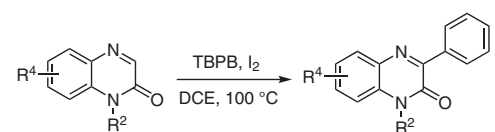

Scheme 4 Studies reported herein

Initially, we chose 1-methylquinoxalin-2-(1H)-one (1a) and acetophenone (2a) as model substrates. The reaction was carried out using $20 \mathrm{~mol} \%$ of TBAI, 5 equivalents of tertbutyl hydroperoxide (TBHP, 70\% solution in water) in DCE at $100{ }^{\circ} \mathrm{C}$. Under these conditions, the desired product 3a was obtained in $32 \%$ yield (Table 1, entry 4 ).

Next, we studied a series of iodides and found that using TBAI as catalyst gave higher yields (Table 1, entries 1-7). Then, several oxidants were investigated, such as dibenzoyl peroxide (BPO), tert-butyl peroxybenzoate (TBPB), dibutyl peroxide, $\mathrm{K}_{2} \mathrm{~S}_{2} \mathrm{O}_{8}$, and DDQ but the reaction with TBHP still resulted in the best result (Table 1, entries 8-11). When we increased the amount of (2a) from 2 equivalents to 4 equivalents and decreased the amount of TBHP from 5 equivalents to 3 equivalents, the yield of target product was increased to $86 \%$ (Table 1, entries 12,13). Finally, other solvents (DMSO, $\mathrm{H}_{2} \mathrm{O}, 1,4$-dioxane, toluene) were studied instead, but unfortunately no improvement in yield was observed (Table 1, entries 14-17). Ultimately, we chose (1a, $0.3 \mathrm{mmol}$ ), (2a, 4.0 equiv.), TBAI (20 mol\%), and TBHP (3.0 equiv.) in DCE ( $2 \mathrm{~mL}$ ) at $100{ }^{\circ} \mathrm{C}$ for 48 hours as the optimal reaction conditions.

Unexpectedly, we observed a byproduct in a relatively low yield, with 3-arylquinoxalin-2(1H)-one 4 a being observed when BPO or TBPB were used as oxidant. It was observed that reaction with iodine showed a slightly higher yield (Table 2, entry 2). Subsequently, a variety of solvents 
Table 1 Optimization of the Reaction Conditions ${ }^{\mathrm{a}}$

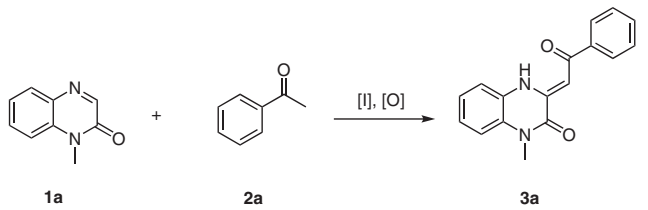

\begin{tabular}{|c|c|c|c|c|c|}
\hline Entry & {$[\mathrm{l}] / \mathrm{mmol} \%$} & [O]/equiv. & Solvent & Temp $\left({ }^{\circ} \mathrm{C}\right)$ & Yield (\%) ${ }^{\mathrm{b}}$ \\
\hline 1 & $\mathrm{I}_{2} / 20$ & TBHP/5 & DCE & 100 & 12 \\
\hline 2 & $\mathrm{Cul} / 20$ & TBHP/5 & DCE & 100 & 24 \\
\hline 3 & $\mathrm{NIS} / 20$ & TBHP/5 & DCE & 100 & NR \\
\hline 4 & TBAI/20 & TBHP/5 & DCE & 100 & 32 \\
\hline 5 & $\mathrm{TBAI}+\mathrm{I}_{2} / 20+10$ & TBHP/5 & DCE & 100 & 23 \\
\hline 6 & TBAI/10 & TBHP/5 & DCE & 100 & 8 \\
\hline 7 & TBAI/100 & TBHP/5 & DCE & 100 & 12 \\
\hline 8 & TBAI/20 & $\mathrm{BPO} / 5$ & DCE & 100 & 25 \\
\hline 9 & TBAI/20 & $\mathrm{K}_{2} \mathrm{~S}_{2} \mathrm{O}_{8} / 5$ & DCE & 100 & NR \\
\hline 10 & $\mathrm{TBAl} / 20$ & $\mathrm{DDQ} / 5$ & DCE & 100 & NR \\
\hline 11 & $\mathrm{TBAl} / 20$ & TBPB $/ 5$ & DCE & 100 & 30 \\
\hline $12^{c}$ & $\mathrm{TBAl} / 20$ & $\mathrm{TBHP} / 5$ & DCE & 100 & 79 \\
\hline $13^{c}$ & $\mathrm{TBAl} / 20$ & $\mathrm{TBHP} / 3$ & DCE & 100 & 86 \\
\hline $14^{c}$ & TBAI/20 & TBHP/3 & dioxane & 100 & 16 \\
\hline $15^{c}$ & $\mathrm{TBAl} / 20$ & $\mathrm{TBHP} / 3$ & DMSO & 100 & trace \\
\hline $16^{c}$ & TBAI/20 & $\mathrm{TBHP} / 3$ & $\mathrm{H}_{2} \mathrm{O}$ & 100 & 58 \\
\hline $17^{c}$ & TBAI/20 & $\mathrm{TBHP} / 3$ & DCE & 90 & 70 \\
\hline $18^{c}$ & TBAI/20 & TBHP/3 & DCE & 110 & 67 \\
\hline
\end{tabular}

${ }^{\text {a }}$ Reaction conditions: $1 \mathbf{a}(0.3 \mathrm{mmol}), \mathbf{2 a}$ ( 2.0 equiv.), solvent $(2 \mathrm{~mL})$, sealed tube, $48 \mathrm{~h}$.

b Isolated yields.

c 1 a $(0.3 \mathrm{mmol})$, 2a (4.0 equiv.).

such as acetonitrile, 1,4-dioxane, and DMSO was investigated (Table 2, entries 6-8). Finally, we surveyed varying the effect of temperature (Table 2, entries 4,5 ), but the reaction did not proceed well. In order to confirm the source of the aryl group, we conducted experiments without acetophenone. To our satisfaction, the yield of $\mathbf{4 a}$ was increased from $34 \%$ to $61 \%$ (Table 2, entry 9). Finally, the number of equivalents of iodine and TBPB was investigated, and the yield was eventually improved to $95 \%$ (Table 2, entries 10-13). Considering the hazards associated with TBPB, 5 equivalents of TBPB were chosen as the preferred conditions. Thus, the optimized reaction conditions were chosen as TBPB (5 equiv.), $\mathrm{I}_{2}\left(2 \mathrm{~mol} \%\right.$ ) in DCE at $100{ }^{\circ} \mathrm{C}$ for 8 hours.

With the optimized conditions in hand, a range of quinoxalin-2(1H)-ones was investigated to give the corresponding derivatives 3 (Scheme 5). These $\mathrm{N}$-substituted quinoxalin-2(1H)-one analogues showed good reactivities, giving the anticipated products 3aa-aj in 32-93\% yields.
Table 2 Optimization of the Reaction Conditions ${ }^{\mathrm{a}}$

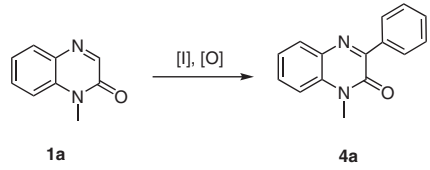

\begin{tabular}{|c|c|c|c|c|c|}
\hline Entry & [I]/mmol\% & [O]/equiv. & Solvent & Temp $\left({ }^{\circ} \mathrm{C}\right)$ & Yield (\%) \\
\hline 1 & TBAI/20 & ТВРВ/5 & DCE & 100 & 30 \\
\hline 2 & $\mathrm{I}_{2} / 20$ & TBPB $/ 5$ & DCE & 100 & 34 \\
\hline 3 & $\mathrm{KI} / 20$ & TВPB $/ 5$ & DCE & 100 & 33 \\
\hline 4 & $\mathrm{I}_{2} / 20$ & TBPB $/ 5$ & DCE & 90 & 25 \\
\hline 5 & $\mathrm{I}_{2} / 20$ & TBPB/5 & DCE & 110 & 28 \\
\hline 6 & $\mathrm{I}_{2} / 20$ & TBPB/5 & $\mathrm{CH}_{3} \mathrm{CN}$ & 100 & 38 \\
\hline 7 & $\mathrm{I}_{2} / 20$ & TBPB/5 & dioxane & 100 & 41 \\
\hline 8 & $\mathrm{I}_{2} / 20$ & TBPB $/ 5$ & DMSO & 100 & NR \\
\hline $9^{c}$ & $\mathrm{I}_{2} / 20$ & ТВРВ $/ 5$ & DCE & 100 & 61 \\
\hline $10^{c}$ & $\mathrm{I}_{2} / 50$ & ТВРВ $/ 5$ & DCE & 100 & 27 \\
\hline $11^{c}$ & $\mathrm{I}_{2} / 2$ & TBPB/5 & DCE & 100 & 88 \\
\hline $12^{c}$ & $\mathrm{I}_{2} / 2$ & ТВРВ/4 & DCE & 100 & 71 \\
\hline $13^{c}$ & $\mathrm{I}_{2} / 2$ & ТВРВ/7 & DCE & 100 & 95 \\
\hline
\end{tabular}

a Reaction conditions: 1 a $(0.3 \mathrm{mmol}), \mathbf{2 a}$ (2.0 equiv.), solvent $(2.0 \mathrm{~mL})$, sealed tube, $48 \mathrm{~h}$.

${ }^{\mathrm{b}}$ Isolated yields.

c Acetophenone was not present.

In particular, an $\mathrm{N}$-phenyl-substituted quinoxalin$2(1 \mathrm{H})$-one was well tolerated, giving the corresponding product 3aj in $64 \%$ yield. Likewise, it was found that substrates with $\mathrm{N}$-propyl, $\mathrm{N}$-butyl and $\mathrm{N}$-cyclohexylmethyl substitution provided the desired products (3ag, 3ae, and 3af) in good yields. Then, we explored substitution at $\mathrm{R}^{1}$ and $\mathrm{R}^{2}$ with $\mathrm{R}^{3}=\mathrm{CH}_{3}$ (Scheme 5). Electron-donating and electron-withdrawing groups provided the desired products in moderate to good yields (3ak, 3al, and 3an). Subsequently, we found that electron-withdrawing substituents $(-\mathrm{F},-\mathrm{Br})$ had a positive effect compared to an electrondonating group $\left(-\mathrm{CH}_{3}\right)$. Furthermore, a substrate with an additional aromatic ring resulted a high yield of 73\% (3am).

Finally, we studied the scope of acetophenones (Scheme 6). When the substrates possessed electron-donating groups, the yields were lower with a substituent at the ortho position than at the para position. In addition, $o$-hydroxyacetophenone also gave the desired product $3 \mathbf{b a}$ in $57 \%$. yield. With electron-withdrawing groups present at the ortho or para positions, the corresponding products $\mathbf{3 b b}$-bi were obtained in $33-74 \%$ yields. The $\pi$-extended aromatic substrate provided the expected product $\mathbf{3 b j}$ in a $78 \%$ yield.

Most of the substrates studied gave the expected products 4aa-ai in moderate to excellent yields (Scheme 7). $\mathrm{N}$-Substituted quinoxalin-2(1H)-ones containing $\mathrm{N}$-ester, $\mathrm{N}$-benzyl, $\mathrm{N}$-benzene acetyl, $\mathrm{N}$-propyl, and $\mathrm{N}$-aryl substitu- 


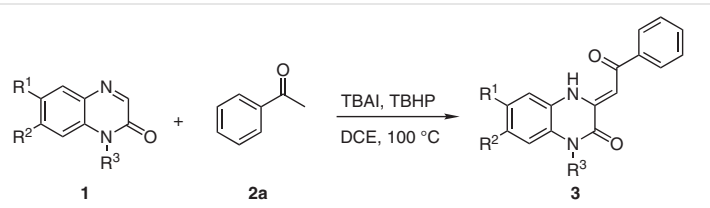<smiles>CCOC(=O)CCN1C(=O)C(=CC(=O)c2ccccc2)Nc2ccccc21</smiles><smiles>CCCN1C(=O)C(=CC(=O)C2CCCC2)Nc2ccccc21</smiles><smiles>C/C=C\C#CC1=CC(=O)N(Cc2ccc(C(C)(C)C)cc2)c2ccccc2N1</smiles><smiles>O=C(/C=C1\Nc2ccccc2N(C/C=C/c2ccccc2)C1=O)c1ccccc1</smiles>

3aa, $46 \%$ $3 a b, 32 \%$ 3ad, $42 \%$<smiles>CCCCN1C(=O)/C(=C/C(=O)c2ccccc2)Nc2ccccc21</smiles>

3ae, $93 \%$<smiles>O=C(/C=C1\Nc2ccccc2N(CC2CCCCC2)C1=O)c1ccccc1</smiles>

3af, $65 \%$

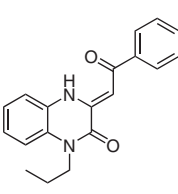

3ag, $63 \%$

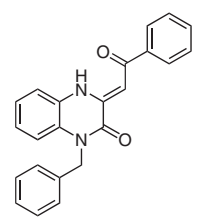

3ah, $69 \%$<smiles>CC=CCCCN1C(=O)C(=CC(=O)c2ccccc2)Nc2ccccc21</smiles>

3ai, $70 \%$

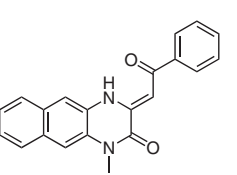

3am, 73\%

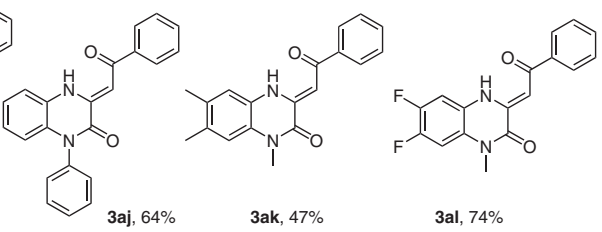<smiles>CN1C(=O)C(=CC(=O)c2ccccc2)C(=O)N=C1c1ccc(Br)cc1</smiles>

3an, $51 \%$

Scheme 5 Substrate scope of quinoxalin-2(1H)-ones. Reagents and conditions: 1 ( $0.3 \mathrm{mmol}), \mathbf{2 a}$ (4.0 equiv.), TBAI (0.2 equiv.), TBHP (3.0 equiv.), DCE ( $2 \mathrm{~mL})$, sealed tube. ${ }^{27}$

ents were all suitable for this reaction, providing the desired products in moderate to good yields. In addition, $\mathrm{N}$ phenyl quinoxalinone gave the corresponding product 4af in $94 \%$ yield. Finally, we explored substitutions at $R^{1}$ and $R^{2}$ with $\mathrm{R}^{3}=\mathrm{CH}_{3}$. An electron-withdrawing substituent $(-\mathrm{Cl})$ had a positive effect compared to an electron-donating group $\left(-\mathrm{CH}_{3}\right)$. Appending an additional aromatic ring also led to 4 ai in $70 \%$ yield (Scheme 7).

Addition of a radical-trapping reagent such as 2,2,6,6tetramethylpiperidine $\mathrm{N}$-oxide (TEMPO) or BHT to the reaction suppressed the transformation, strongly indicating that the $\mathrm{C}-\mathrm{C}$ bond formation is a radical-mediated process (Scheme 8).

On the basis of this result, a plausible reaction mechanism can be proposed (Scheme 9). Either the tert-butoxy radical or tert-butylhydroperoxy radical can remove a hydrogen atom from $\mathbf{2 a}$ to form radical intermediate $\mathbf{5}$. Addition of intermediate $\mathbf{5}$ to $\mathbf{1 a}$ affords intermediate $\mathbf{6}$. Then

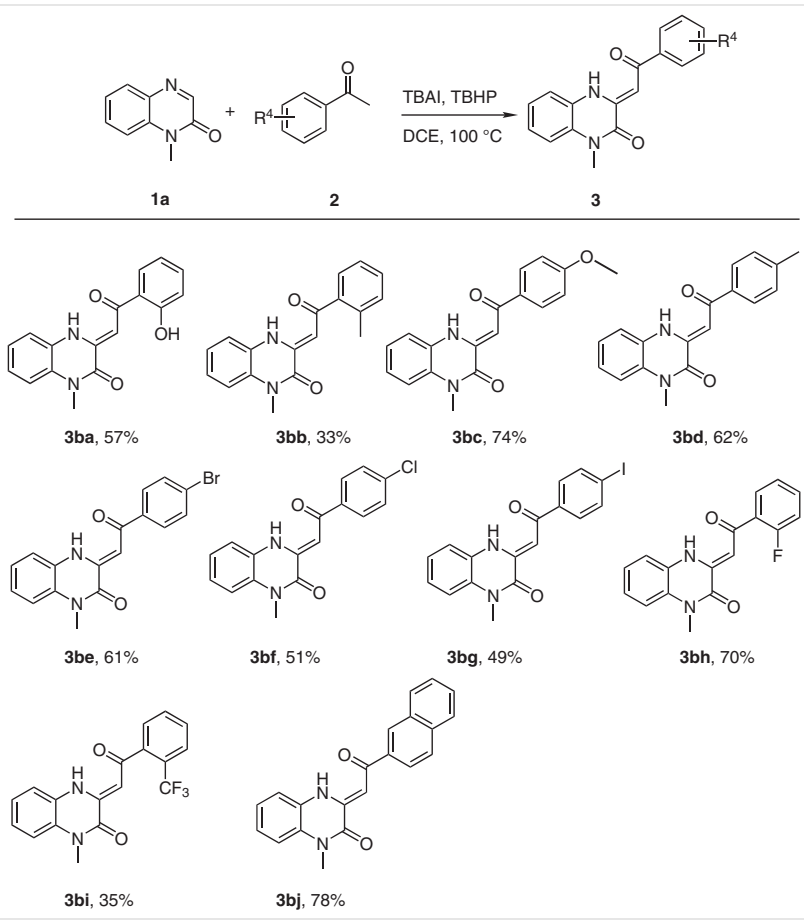

Scheme 6 Substrate scope of quinoxalin-2(1H)-ones. Reagents and conditions: 1 a ( $0.3 \mathrm{mmol}), \mathbf{2}$ (4.0 equiv.), TBAI (0.2 equiv.), TBHP (3.0 equiv.), DCE ( $2 \mathrm{~mL})$, sealed tube. ${ }^{27}$

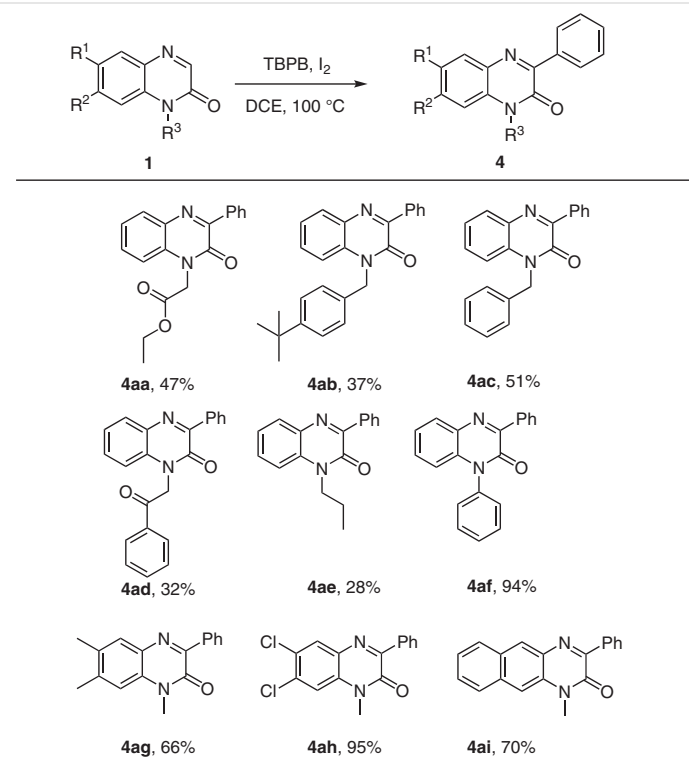

Scheme 7 Substrate scope of quinoxalin-2(1H)-ones. Reagents and conditions: 1 a ( $0.3 \mathrm{mmol}), \mathrm{I}_{2}$ (0.02 equiv.), TBPB (5.0 equiv.), solvent $(2.0 \mathrm{~mL})$, sealed tube. ${ }^{27}$

intermediate 6 can undergo 1,2-H shift to form the more stable intermediate $\mathbf{7}$. Finally, the final product $\mathbf{3 a}$ is obtained by hydrogen-atom removal by a tert-butoxy radical. 
(a)

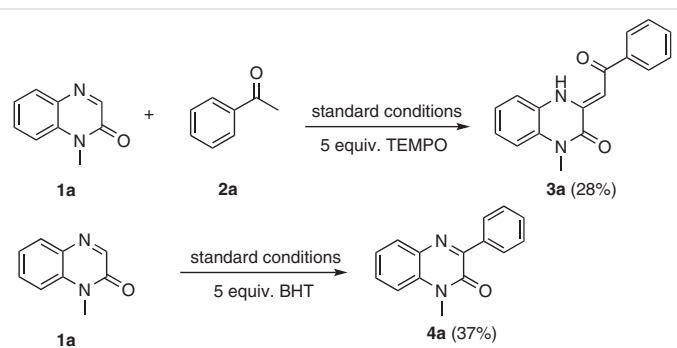

Scheme 8 Experiments with added radical inhibitors
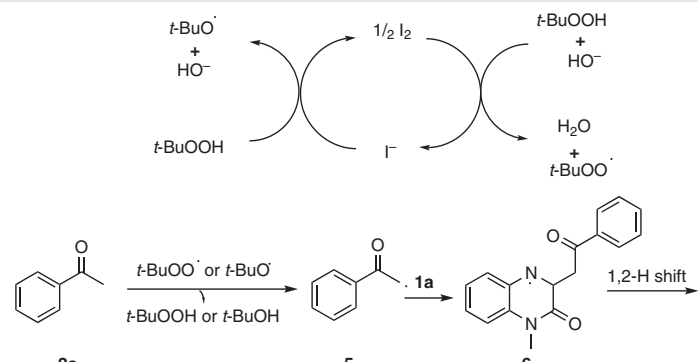

2a

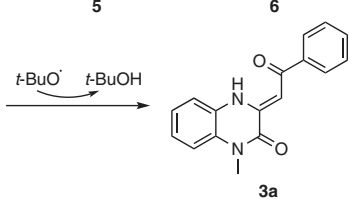

Scheme 9 Plausible reaction mechanism

For the TBPB reaction catalyzed by $\mathrm{I}_{2}$, the benzoyl radical releases carbon dioxide forming the phenyl radical under standard conditions. Addition of the phenyl radical to the carbon-nitrogen double bond affords radical intermediate 10, which is further oxidized by the iodide cation to form nitrogen cation compound $\mathbf{1 1}$ that then undergoes 1,2-H shift to give 12. Finally, the desired compound is obtained by hydrogen-atom removal to give $\mathbf{4 a}$ (Scheme 10).

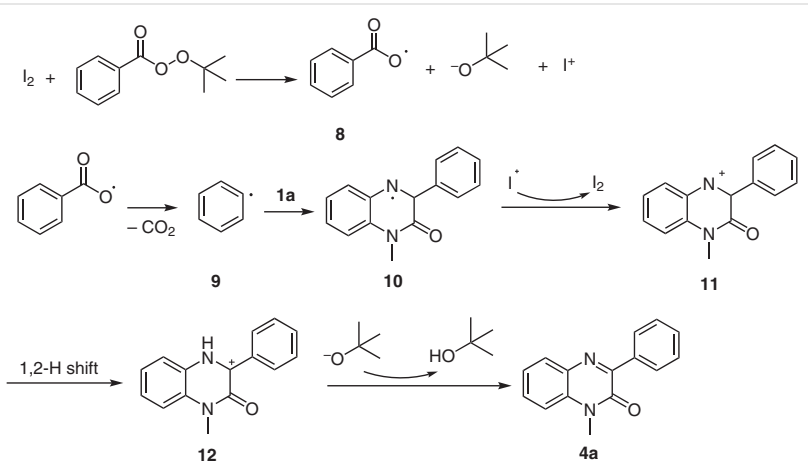

Scheme 10 Plausible reaction mechanism

In conclusion, we have developed a novel protocol for direct synthesis of 3-(2-oxo-2-phenylethylidene)-3,4-dihydroquinoxalin-2(1H)-ones. The iodide/peroxide system has been shown to be a powerful combination to activate the $\mathrm{C}-\mathrm{H}$ bond of quinoxalin-2(1H)-ones. This process exhibits good functional group tolerance with a broad substrate scope, resulting in acetylation of quinoxalin-2(1H)-ones and providing a new method for the introduction of an aryl group.

\section{Conflict of Interest}

The authors declare no conflict of interest.

\section{Funding Information}

Financial support from the National Science Foundation of China (21572117) and the Shandong Key Research Program (Nos. 2019JZZY021015 and 2019GHY112053) is gratefully acknowledged.

\section{Acknowledgment}

We are grateful to the Analytical Center for Structural Constituent and Physical Property of Core Facilities Sharing Platform, Shandong University for their technological and service support.

\section{Supporting Information}

Supporting information for this article is available online at https://doi.org/10.1055/a-1489-8711.

\section{References and Notes}

(1) El-Hawash, S. A. M.; Habi, N. S.; Kassem, M. A. Arch. Pharm. Chem. Life Sci. 2006, 339, 564.

(2) Qin, X.; Hao, X.; Han, H.; Zhu, S.; Yang, Y.; Wu, B.; Hussain, S.; Parveen, S.; Jing, C.; Ma, B.; Zhu, C. J. Med. Chem. 2015, 58, 1254.

(3) Ahmed, H. E. A.; Ihmaid, S. K.; Omar, A. M.; Shehata, A. M.; Rateb, H. S.; Zayed, M. F.; Ahmed, S.; Elaasser, M. M. Bioorg. Chem. 2018, 76, 332.

(4) Koltun, D. O.; Parkhill, E. Q.; Vasilevich, N. I.; Glushkov, A. I.; Zilbershtein, A. I.; Ivanov, A. V.; Cole, A. G.; Henderson, I.; Zautke, N. A.; Brunn, S. A.; Mollova, N.; Leung, K.; Chisholm, J. W.; Zablocki, J. Bioorg. Med. Chem. Lett. 2009, 19, 2048.

(5) Shawali, A. S.; Zayed, M. M.; Farghaly, T. A. J. Heterocycl. Chem. 2005, 42, 185.

(6) Wu, B.; Yang, Y.; Qin, X.; Zhang, S.; Jing, C.; Zhu, C.; Ma, B. Chem. Med. Chem. 2013, 8, 1913.

(7) (a) Křupková, S.; Funk, P.; Soural, M.; Hlaváč, J. ACS Comb.Sci. 2013, 15, 20. (b) Paul, S.; Khanal, H. D.; Clinton, C. D.; Kim, S. H.; Lee, Y. R. Org. Chem. Front. 2019, 6, 231. (c) Ramesh, B.; Reddy, C. R.; Kumar, G. R.; Reddy, B. V. S. Tetrahedron Lett. 2018, 59, 628. (d) Yin, K.; Zhang, R. Org. Lett. 2017, 19, 1530. (e) Paul, S.; Ha, J. H.; Park, G. E.; Lee, Y. R. Adv. Synth. Catal. 2017, 359, 1515.

(8) Fu, J.; Yuan, J.; Zhang, Y.; Xiao, Y.; Mao, P.; Diao, X.; Qu, L. Org. Chem. Front. 2018, 5, 3382.

(9) Zhou, J.; Zhou, P.; Zhao, T.; Ren, Q.; Li, J. Adv. Synth. Catal. 2019, $361,5371$.

(10) Chu, X.; Wu, Y.; Lu, H.; Yang, B.; Ma, C. Eur. J. Org. Chem. 2020, 1141. 
(11) Li, K.-J.; Jiang, Y.-Y.; Xu, K.; Zeng, C.-C.; Sun, B.-G. Green Chem. 2019, 21, 4412

(12) Wang, J.; Sun, B.; Zhang, L.; Xu, T.; Xie, Y.; Jin, C. Org. Chem. Front. 2020, 7, 113.

(13) (a) Gupta, A.; Deshmukh, M. S.; Jain, N. J. Org. Chem. 2017, 82, 4784. (b) Wei, W.; Wang, L.; Bao, P.; Shao, Y.; Yue, H.; Yang, D.; Yang, X.; Zhao, X.; Wang, H. Org. Lett. 2018, 20, 7125. (c) Yang, Q.; Yang, Z.; Tan, Y.; Zhao, J.; Sun, Q.; Zhang, H.-Y.; Zhang, Y. Adv. Synth. Catal. 2019, 361, 1662.

(14) Wang, L.; Zhang, Y.; Li, F.; Li, X.; Hao, H.-Y.; Zhang, J. Adv. Synth. Catal. 2018, 360, 3969.

(15) Petronijević, J.; Bugarčić, Z.; Bogdanović, G. A.; Stefanović, S.; Janković, N. Green Chem. 2017, 19, 707.

(16) Beletskaya, I. P.; Cheprakov, A. V. Chem. Rev. 2000, 100, 3009.

(17) Gandeepan, P.; Koeller, J.; Korvorapun, K.; Mohr, J.; Ackermann, L. Angew. Chem. Int. Ed. 2019, 58, 982.

(18) Yi, H.; Zhang, G.; Wang, H.; Huang, Z.; Wang, J.; Singh, A. K.; Lei, A. Chem. Rev. 2017, 117, 9016.

(19) Chen, L.; Shi, E.; Liu, Z.; Chen, S.; Wei, W.; Li, H.; Xu, K.; Wan, X. Chem. Eur. J. 2011, 17, 4085.

(20) Bozdyreva, K. S.; Smirnova, I. V.; Maslivets, A. N. Russ. J. Org. Chem. 2005, 41, 1081.

(21) Dounay, A. B.; Overman, L. E. Chem. Rev. 2003, 103, 2945.

(22) (a) Shen, Z.; Huang, H.; Zhu, C.; Warratz, S.; Ackermann, L. Org. Lett. 2019, 21, 571. (b) Shen, H.; Deng, Q.; Liu, R.; Feng, Y.; Zheng, C.; Xiong, Y. Org. Chem. Front. 2017, 4, 1806.

(23) Yadav, D. K. T.; Bhanage, B. M. Synlett 2015, 26, 1862.

(24) Zhang, F.; Li, L. S. Zhang J. Y.; Gong, H. Sci. Rep. 2019, 9, 2787.

(25) Yi, H.; Zhang, G. T.; Wang, H. M.; Huang, Z. Y.; Wang, J.; Singh, A. K.; Lei, A. Chem. Rev. 2017, 117, 9016.

(26) Chen, L.; Shi, Er. B.; Liu, Z. J.; Chen, S. L.; Wei, W.; Li, H.; Xu, K.; Wan, X. B. Chem. Eur. J. 2011, 17, 4085.

(27) Substituted substrates 1 were obtained according to the literature reports. ${ }^{28}$ Other reagents and solvents were obtained from commercial available reagents and solvents and were used directly without further purification. All the reactions were monitored by thin-layer chromatography. ${ }^{1} \mathrm{H}$ NMR spectra were recorded on a Bruker Avance 500 spectrometer at $500 \mathrm{MHz}$ using $\mathrm{CDCl}_{3}$ or DMSO- $d_{6}$ as solvent and tetramethylsilane (TMS) as internal standard. ${ }^{13} \mathrm{C}$ NMR spectra were run in the same instrument at $125 \mathrm{MHz}$. HRMS spectra were measured on a QTOF instrument in positive-ion mode with an ESI ion source. Melting points were recorded on an XD-4 digital micro melting point apparatus.

\section{General Procedure for the TBAI-Catalyzed C-H Acetylation}

A mixture of quinoxalin-2(1H)-one $(\mathbf{1}, 0.3 \mathrm{mmol})$, acetophenone ( $2,4.0$ equiv.), TBAI ( 0.2 equiv.), and TBHP (3.0 equiv.) in DCE $(2.0 \mathrm{~mL})$ in a sealed tube was heated at $100{ }^{\circ} \mathrm{C}$ for $48 \mathrm{~h}$. After completion of the reaction, the tube was then cooled to room temperature and extracted with ethyl acetate $(3 \times 20 \mathrm{~mL})$. The combined organic phases were dried over anhydrous $\mathrm{Na}_{2} \mathrm{SO}_{4}$, filtered, and concentrated in vacuo. The residue was purified by column chromatography (petroleum ether/ethyl acetate $=30: 1$ ) on silica gel to provide pure product.

\section{General Procedure for the $\mathbf{I}_{\mathbf{2}}$-Catalyzed C-H Arylation}

A mixture of quinoxalin-2(1H)-one $(\mathbf{1}, 0.3 \mathrm{mmol}), \mathrm{I}_{2}(0.02$ equiv), and TBPB (5.0 equiv.) in DCE solvent $(2.0 \mathrm{~mL}$ ) in a sealed tube was heated at $100{ }^{\circ} \mathrm{C}$ for $6 \mathrm{~h}$. After completion of the reaction, the tube was then cooled to room temperature and extracted with ethyl acetate $(3 \times 20 \mathrm{~mL})$. The combined organic phases were dried over anhydrous $\mathrm{Na}_{2} \mathrm{SO}_{4}$, filtered, and concentrated in vacuo. The residue was purified by column chromatog- raphy (petroleum ether/ethyl acetate $=10: 1$ ) on silica gel to provide the pure product.

(Z)-1-Methyl-3-(2-oxo-2-phenylethylidene)-3,4-dihydroquinoxalin-2(1H)-one (3a)

Yield 86\%; yellow solid; mp $176-181{ }^{\circ} \mathrm{C} .{ }^{1} \mathrm{H}$ NMR $(500 \mathrm{MHz}$, $\left.\mathrm{CDCl}_{3}\right): \delta=14.01(\mathrm{~s}, 1 \mathrm{H}), 8.13(\mathrm{~d}, J=7.3 \mathrm{~Hz}, 2 \mathrm{H}), 8.03(\mathrm{~d}, J=7.1$ $\mathrm{Hz}, 2 \mathrm{H}$ ), 7.51-7.47 (m, $4 \mathrm{H}$ ), 7.18 (s, $2 \mathrm{H}$ ), 7.01 (s, $1 \mathrm{H}$ ), 3.65 (s, 1 H). ${ }^{13} \mathrm{C}$ NMR $\left(500 \mathrm{MHz}, \mathrm{CDCl}_{3}\right): \delta=190.2,156.3,144.6,138.9$, 131.9, 130.2, 128.5, 127.5, 125.3, 124.4, 124.1, 116.8, 114.4, 91.0, 29.9. HRMS (ESI): $m / z[M+H]^{+}$calcd for $\mathrm{C}_{17} \mathrm{H}_{14} \mathrm{~N}_{2} \mathrm{O}_{2}$ : 279.1134; found: 279.1135 .

Ethyl-(E)-4-[(Z)-2-oxo-3-(2-oxo-2-phenylethylidene)-3,4dihydroquinoxalin-1(2H)-yl]but-2-enoate (3aa)

Yield 46\%; yellow solid; mp $167-170{ }^{\circ} \mathrm{C} .{ }^{1} \mathrm{H}$ NMR $(500 \mathrm{MHz}$, DMSO- $\left.d_{6}\right): \delta=13.82(\mathrm{~s}, 1 \mathrm{H}), 8.00(\mathrm{~d}, J=7.3 \mathrm{~Hz}, 2 \mathrm{H}), 7.60-7.52$ $(\mathrm{m}, 4 \mathrm{H}), 7.28(\mathrm{~d}, J=9.0 \mathrm{~Hz}, 1 \mathrm{H}), 7.24-7.19(\mathrm{~m}, 2 \mathrm{H}), 7.04\left(\mathrm{dt}, J_{1}=\right.$ $\left.15.9 \mathrm{~Hz}, J_{2}=4.3 \mathrm{~Hz}, 1 \mathrm{H}\right), 6.87(\mathrm{~s}, 1 \mathrm{H}), 5.96(\mathrm{~d}, J=15.9 \mathrm{~Hz}, 1 \mathrm{H})$, $5.02(\mathrm{~s}, 2 \mathrm{H}), 4.11(\mathrm{q}, J=7.1 \mathrm{~Hz}, 2 \mathrm{H}), 1.18(\mathrm{t}, J=7.1 \mathrm{~Hz}, 3 \mathrm{H}) .{ }^{13} \mathrm{C}$ NMR $\left(125 \mathrm{MHz}\right.$, DMSO- $\left.d_{6}\right): \delta=188.7,165.6,156.0,145.2,142.7$, $139.1,132.4,129.2,127.7,127.4,125.5,124.6,122.1,117.7$, $115.7,90.1,60.5,40.5,14.5$. HRMS (ESI)): $\mathrm{m} / z[\mathrm{M}+\mathrm{H}]^{+}$calcd for $\mathrm{C}_{22} \mathrm{H}_{20} \mathrm{~N}_{2} \mathrm{O}_{4}$ : 377.1501; found: 377.1502.

(Z)-4-[2-0xo-3-(2-oxo-2-phenylethylidene)-3,4-dihydroquinoxalin-1(2H)-yl]butanoate (3ab)

Yield 32\%; yellow solid; mp 256-260 ${ }^{\circ} \mathrm{C} .{ }^{1} \mathrm{H}$ NMR (400 MHz, DMSO- $\left._{6}\right): \delta=13.85(\mathrm{~s}, 1 \mathrm{H}), 7.99(\mathrm{~d}, J=5.9 \mathrm{~Hz}, 2 \mathrm{H}), 7.59-7.54$ (m, $7 \mathrm{H}), 7.28-7.22(\mathrm{~m}, 2 \mathrm{H}), 6.86(\mathrm{~s}, 1 \mathrm{H}), 4.25-4.22(\mathrm{~m}, 2 \mathrm{H})$, 4.06-4.04 (m, $2 \mathrm{H}), 2.51-2.49$ (m, $2 \mathrm{H}), 1.95(\mathrm{t}, J=5.8 \mathrm{~Hz}, 3 \mathrm{H})$. ${ }^{13} \mathrm{C}$ NMR $\left(100 \mathrm{MHz}\right.$, DMSO- $\left.d_{6}\right): \delta=188.6,172.9,155.8,145.1$, 139.0, 132.4, 129.2, 127.8, 127.4, 125.3, 124.8, 124.5, 117.9, 115.3, 89.9, 60.4, 31.1, 22.3, 14.5. HRMS (ESI): $m / z[\mathrm{M}+\mathrm{H}]^{+}$ calcd for $\mathrm{C}_{22} \mathrm{H}_{22} \mathrm{~N}_{2} \mathrm{O}_{4}$ : 379.1658; found: 379.1653.

(Z)-1-[4-(tert-Butyl)benzyl]-3-(2-oxo-2-phenylethylidene)3,4-dihydroquinoxalin-2(1H)-one (3ac)

Yield 76\%; yellow solid; mp $188-190{ }^{\circ} \mathrm{C} .{ }^{1} \mathrm{H}$ NMR $(500 \mathrm{MHz}$, DMSO- $\left._{6}\right): \delta=13.86(\mathrm{~s}, 1 \mathrm{H}), 8.01(\mathrm{~d}, J=7.6 \mathrm{~Hz}, 2 \mathrm{H}), 7.57-7.53$ $(\mathrm{m}, 6 \mathrm{H}), 7.33-7.24(\mathrm{~m}, 5 \mathrm{H}), 6.94(\mathrm{~s}, 1 \mathrm{H}), 5.43(\mathrm{~s}, 2 \mathrm{H}), 1.22(\mathrm{~s}, 9$ H). ${ }^{13} \mathrm{C}$ NMR (101 MHz, DMSO- $\left.d_{6}\right): \delta=188.7,156.0,150.6$, $145.1,139.0,133.1,132.5,129.3,127.9,127.5,127.0,125.9$, 125.5, 124.7, 117.8, 116.02, 90.29, 45.6, 34.7, 31.6. HRMS (ESI): $m / z[\mathrm{M}+\mathrm{H}]^{+}$calcd for $\mathrm{C}_{22} \mathrm{H}_{20} \mathrm{~N}_{2} \mathrm{O}_{4}$ ?411.2073; found: 411.2071 . (Z)-1-Cinnamyl-3-(2-oxo-2-phenylethylidene)-3,4-dihydroquinoxalin-2(1H)-one (3ad)

Yield 42\%; yellow solid; mp 204-207 ${ }^{\circ} \mathrm{C} .{ }^{1} \mathrm{H}$ NMR $(500 \mathrm{MHz}$, DMSO- $\left.d_{6}\right): \delta=13.86(\mathrm{~s}, 1 \mathrm{H}), 8.00(\mathrm{~d}, J=7.3 \mathrm{~Hz}, 2 \mathrm{H}), 7.59-7.52$ (m, $5 \mathrm{H}), 7.43-7.40(\mathrm{~m}, 3 \mathrm{H}), 7.31(\mathrm{t}, J=7.4 \mathrm{~Hz}, 2 \mathrm{H}), 7.23-7.21$ $(\mathrm{m}, 2 \mathrm{H}), 6.90(\mathrm{~s}, 1 \mathrm{H}), 5.01(\mathrm{~s}, 2 \mathrm{H}), 2.50(\mathrm{~s}, 2 \mathrm{H}) .{ }^{13} \mathrm{C}$ NMR $(125$ MHz, DMSO- $\left.d_{6}\right): \delta=188.7,155.8,145.2,139.1,136.6,132.4$, $132.1,129.3,129.1,128.2,127.9,127.5,126.8,125.4,124.7$, 124.6, 123.7, 117.7, 115.9, 44.7. HRMS (ESI): $m / z[\mathrm{M}+\mathrm{H}]^{+}$calcd for $\mathrm{C}_{25} \mathrm{H}_{20} \mathrm{~N}_{2} \mathrm{O}_{2}$ : 381.1603; found: 381.1603 .

(Z)-1-Butyl-3-(2-oxo-2-phenylethylidene)-3,4-dihydroquinoxalin-2(1H)-one (3ae)

Yield 93\%; colorless oily liquid. ${ }^{1} \mathrm{H}$ NMR $\left(500 \mathrm{MHz}\right.$, DMSO- $d_{6}$ ): $\delta=14.06(\mathrm{~s}, 1 \mathrm{H}), 8.04-8.03(\mathrm{~m}, 2 \mathrm{H}), 7.52-7.45(\mathrm{~m}, 3 \mathrm{H}), 7.23-$ 7.18 (m, $4 \mathrm{H}), 7.02(\mathrm{~s}, 1 \mathrm{H}), 1.24-1.20(\mathrm{~m}, 2 \mathrm{H}), 1.80-1.72(\mathrm{~m}, 2$ $\mathrm{H}), 1.55-1.45(\mathrm{~m}, 2 \mathrm{H}), 1.00(\mathrm{t}, J=7.3 \mathrm{~Hz}, 3 \mathrm{H}) .{ }^{13} \mathrm{C}$ NMR $(101$ $\left.\mathrm{MHz}, \mathrm{CDCl}_{3}\right): \delta=190.0,156.0,144.7,139.8,131.8,128.5,127.4$, $125.5,124.2,124.0,117.1,114.5,90.8,77.4,77.0,76.7,42.7$, 29.1, 20.3, 13.8. HRMS (ESI): $m / z[\mathrm{M}+\mathrm{H}]^{+}$calcd for $\mathrm{C}_{20} \mathrm{H}_{22} \mathrm{~N}_{2} \mathrm{O}$ : 321.1603; found: 321.1602 . 
(Z)-1-(Cyclohexylmethyl)-3-(2-oxo-2-phenylethylidene)-3,4dihydroquinoxalin-2(1H)-one (3af)

Yield $65 \%$; colorless oily liquid. ${ }^{1} \mathrm{H}$ NMR ( $500 \mathrm{MHz}$, DMSO- $d_{6}$ ): $\delta=13.85(\mathrm{~s}, 1 \mathrm{H}), 7.95(\mathrm{~d}, J=7.3 \mathrm{~Hz}, 2 \mathrm{H}), 7.55-7.49(\mathrm{~m}, 4 \mathrm{H})$, $7.39(\mathrm{~d}, J=8 \mathrm{~Hz}, 1 \mathrm{H}), 7.22-7.17(\mathrm{~m}, 2 \mathrm{H}), 6.83(\mathrm{~s}, 1 \mathrm{H}), 4.05(\mathrm{~s}, 2$ $\mathrm{H}), 1.79(\mathrm{~s}, 1 \mathrm{H}), 1.63(\mathrm{~d}, J=8.15 \mathrm{~Hz}, 4 \mathrm{H}), 1.10-1.08(\mathrm{~m}, 6 \mathrm{H}) .{ }^{13} \mathrm{C}$ $\operatorname{NMR}\left(125 \mathrm{MHz}, \mathrm{DMSO}-d_{6}\right): \delta=188.5,156.0,145.0,139.0,132.3$, 129.1, 128.0, 127.4, 125.2, 124.7, 124.4, 117.8, 115.7, 90.0, 48.0, 36.2, 30.6, 26.3, 25.8. HRMS (ESI): $\mathrm{m} / z[\mathrm{M}+\mathrm{H}]^{+}$calcd for $\mathrm{C}_{23} \mathrm{H}_{24} \mathrm{~N}_{2} \mathrm{O}_{2}$ : 361.1916; found: 361.1916 .

(Z)-3-(2-0xo-2-phenylethylidene)-1-propyl-3,4-dihydroquinoxalin-2(1H)-one (3ag)

Yield $63 \%$; colorless oily liquid. ${ }^{1} \mathrm{H}$ NMR (500 MHz, DMSO- $d_{6}$ ): $\delta=13.82(\mathrm{~s}, 1 \mathrm{H}), 7.94(\mathrm{~d}, J=7.1 \mathrm{~Hz}, 2 \mathrm{H}), 7.56-7.54(\mathrm{~m}, 2 \mathrm{H})$, 7.51-7.48 (m, $3 \mathrm{H}), 7.41$ (d, $J=7.8 \mathrm{~Hz}, 1 \mathrm{H}), 7.23-7.16(\mathrm{~m}, 2 \mathrm{H})$, $6.81(\mathrm{~s}, 1 \mathrm{H}), 1.68(\mathrm{q}, J=7.6 \mathrm{~Hz}, 2 \mathrm{H}), 0.96(\mathrm{t}, J=7.4 \mathrm{~Hz}, 3 \mathrm{H}) .{ }^{13} \mathrm{C}$ $\operatorname{NMR}\left(125 \mathrm{MHz}\right.$, DMSO- $\left.d_{6}\right): \delta=188.5,155.6,145.0,139.0,132.3$, 129.1, 127.7, 127.4, 125.2, 124.7, 124.4, 117.8, 115.4, 89.9, 44.1, 20.5, 11.5. HRMS (ESI): $m / z[M+H]^{+}$calcd for $\mathrm{C}_{19} \mathrm{H}_{18} \mathrm{~N}_{2} \mathrm{O}_{2}$ : 307.1447; found: 307.1448 .

(Z)-1-Benzyl-3-(2-oxo-2-phenylethylidene)-3,4-dihydroquinoxalin-2(1H)-one (3ah)

Yield 69\%; yellow solid; mp $180-184{ }^{\circ} \mathrm{C} .{ }^{1} \mathrm{H}$ NMR $(500 \mathrm{MHz}$, DMSO- $\left.d_{6}\right): \delta=13.86(\mathrm{~s}, 1 \mathrm{H}), 8.01(\mathrm{~d}, J=7.1 \mathrm{~Hz}, 2 \mathrm{H}), 7.59-7.54$ (m, $4 \mathrm{H}), 7.34(\mathrm{~d}, J=4.4 \mathrm{~Hz}, 4 \mathrm{H}), 7.27-7.25(\mathrm{~m}, 2 \mathrm{H}), 7.20$ (t, $J=$ $6.7 \mathrm{~Hz}, 1 \mathrm{H}), 7.14(\mathrm{t}, J=7.1 \mathrm{~Hz}, 1 \mathrm{H}), 6.84(\mathrm{~s}, 1 \mathrm{H}), 5.48(\mathrm{~s}, 2 \mathrm{H})$. ${ }^{13} \mathrm{C}$ NMR $\left(125 \mathrm{MHz}\right.$, DMSO- $\left.d_{6}\right): \delta=188.8,156.4,145.1,139.0$, 136.2, 132.5, 129.3, 129.2, 127.8, 127.5, 127.2, 125.4, 124.7, 124.6, 117.8, 116.0, 90.4, 46.1. HRMS (ESI): $\mathrm{m} / z[\mathrm{M}+\mathrm{H}]^{+}$calcd for $\mathrm{C}_{23} \mathrm{H}_{18} \mathrm{~N}_{2} \mathrm{O}_{2}$ : 355.1447; found: 355.1446 .

(Z)-2-[2-0xo-3-(2-oxo-2-phenylethylidene)-3,4-dihydroquinoxalin-1(2H)-yl]ethyl acetate (3ai)

Yield 70\%; yellow solid. ${ }^{1} \mathrm{H}$ NMR ( $500 \mathrm{MHz}$, DMSO- $d_{6}$ ): $\delta=13.72$ $(\mathrm{s}, 1 \mathrm{H}), 8.00(\mathrm{~d}, J=7.1 \mathrm{~Hz}, 2 \mathrm{H}), 7.60-7.57(\mathrm{~m}, 2 \mathrm{H}), 7.55-7.52$ $(\mathrm{m}, 2 \mathrm{H}), 7.37$ (d, $J=9.3 \mathrm{~Hz}, 1 \mathrm{H}), 7.25-7.19(\mathrm{~m}, 2 \mathrm{H}), 6.86(\mathrm{~s}, 1$ H), $5.07(\mathrm{~s}, 2 \mathrm{H}), 4.22(\mathrm{q}, J=7.1 \mathrm{~Hz}, 2 \mathrm{H}), 1.25(\mathrm{t}, J=7.1 \mathrm{~Hz}, 3 \mathrm{H})$. ${ }^{13} \mathrm{C}$ NMR $\left(125 \mathrm{MHz}\right.$, DMSO- $\left.d_{6}\right): \delta=188.9,168.0,156.1,144.3$, 138.9, 132.5, 129.2, 127.8, 127.5, 125.1, 124.8, 124.7, 117.8, 115.3, 90.3, 61.9, 44.7, 14.5. HRMS (ESI): $\mathrm{m} / z[\mathrm{M}+\mathrm{H}]^{+}$calcd for $\mathrm{C}_{20} \mathrm{H}_{18} \mathrm{~N}_{2} \mathrm{O}_{4}$ : 351.1345 ; found: 351.1342 .

(Z)-3-(2-0xo-2-phenylethylidene)-1-phenyl-3,4-dihydroquinoxalin-2(1H)-one (3aj)

Yield 64\%; yellow solid; mp 191-193 ${ }^{\circ} \mathrm{C} .{ }^{1} \mathrm{H}$ NMR (500 MHz, DMSO- $\left.d_{6}\right): \delta=13.86(\mathrm{~s}, 1 \mathrm{H}), 7.99(\mathrm{~d}, J=7.3 \mathrm{~Hz}, 2 \mathrm{H}), 7.67-7.63$ (m, $3 \mathrm{H}), 7.61-7.57$ ( $\mathrm{m}, 3 \mathrm{H}), 7.55-7.52(\mathrm{~m}, 2 \mathrm{H}), 7.46$ (d, $J=7.3$ $\mathrm{Hz}, 2 \mathrm{H}), 7.20(\mathrm{t}, J=7.9 \mathrm{~Hz}, 1 \mathrm{H}), 7.05(\mathrm{t}, J=7.4 \mathrm{~Hz}, 1 \mathrm{H}), 6.88(\mathrm{~s}, 1$ H). ${ }^{13} \mathrm{C}$ NMR $\left(125 \mathrm{MHz}\right.$, DMSO- $\left.d_{6}\right): \delta=189.0,155.9,145.7$, 139.0, 136.9, 132.5, 130.6, 130.1, 129.7, 129.3, 129.2, 127.5, 125.1, 124.5, 124.3, 117.5, 116.0, 90.1. HRMS (ESI): $\mathrm{m} / z[\mathrm{M}+\mathrm{H}]^{+}$ calcd for $\mathrm{C}_{22} \mathrm{H}_{16} \mathrm{~N}_{2} \mathrm{O}_{4}$ : 341.1290; found: 341.1286 .

(Z)-1,6,7-Trimethyl-3-(2-oxo-2-phenylethylidene)-3,4-dihydroquinoxalin-2(1H)-one (3ak)

Yield 47\%; yellow solid; mp 221-224 ${ }^{\circ} \mathrm{C} .{ }^{1} \mathrm{H}$ NMR $(500 \mathrm{MHz}$, $\left.\mathrm{CDCl}_{3}\right): \delta=14.16(\mathrm{~s}, 1 \mathrm{H}), 8.03-8.01(\mathrm{~m}, 2 \mathrm{H}), 7.50-7.46(\mathrm{~m}, 3$ H), $7.01(\mathrm{~s}, 1 \mathrm{H}), 6.97(\mathrm{~s}, 1 \mathrm{H}), 6.95(\mathrm{~s}, 1 \mathrm{H}), 3.64(\mathrm{~s}, 3 \mathrm{H}), 2.31(\mathrm{~s}, 3$ $\mathrm{H}), 2.28(\mathrm{~s}, 3 \mathrm{H}) \cdot{ }^{13} \mathrm{C}$ NMR $\left(125 \mathrm{MHz}, \mathrm{CDCl}_{3}\right): \delta=189.2,156.2$, $145.0,139.0,133.1,131.5,130.1,128.5,127.3,126.5,123.2$, 117.8, 115.3, 90.4, 29.6, 19.9, 19.3. HRMS (ESI): $m / z\left[M+\mathrm{H}^{+}\right.$ calcd for $\mathrm{C}_{19} \mathrm{H}_{18} \mathrm{~N}_{2} \mathrm{O}_{2}$ : 307.1447; found: 306.1445 .

(Z)-6,7-Difluoro-1-methyl-3-(2-oxo-2-phenylethylidene)-

3,4-dihydroquinoxalin-2(1H)-one (3al)

Yield 74\%; yellow solid; mp $286-288{ }^{\circ} \mathrm{C} .{ }^{1} \mathrm{H}$ NMR $(500 \mathrm{MHz}$, $\left.\mathrm{CDCl}_{3}\right): \delta=14.07(\mathrm{~s}, 1 \mathrm{H}), 8.00(\mathrm{~d}, J=7.2 \mathrm{~Hz}, 2 \mathrm{H}), 7.54-7.45(\mathrm{~m}$,
$4 \mathrm{H}), 7.10-7.07(\mathrm{~m}, 1 \mathrm{H}), 7.00(\mathrm{~s}, 1 \mathrm{H}), 3.61(\mathrm{~s}, 3 \mathrm{H}) .{ }^{13} \mathrm{C}$ NMR $\left(125 \mathrm{MHz}, \mathrm{CDCl}_{3}\right): \delta=188.9,155.8,145.0,138.2,132.1,130.1$, 128.6, 127.4, 106.1, 105.9, 103.8, 91.7, 30.2. HRMS (ESI): $m / z$ [M $+\mathrm{H}]^{+}$calcd for $\mathrm{C}_{17} \mathrm{H}_{12} \mathrm{~F}_{2} \mathrm{~N}_{2} \mathrm{O}_{2}$ : 315.0945; found: 315.0940 . (Z)-1-Methyl-3-(2-oxo-2-phenylethylidene)-3,4-dihydrobenzo[g]quinoxalin-2(1H)-one (3am)

Yield 73\%; yellow solid; mp $186-190{ }^{\circ} \mathrm{C} .{ }^{1} \mathrm{H}$ NMR $(500 \mathrm{MHz}$, DMSO- $\left._{6}\right): \delta=13.74(\mathrm{~s}, 1 \mathrm{H}), 8.01-7.98,(\mathrm{~m}, 3 \mathrm{H}), 7.94-7.92(\mathrm{~m}$, $1 \mathrm{H}), 7.85$ (s, $1 \mathrm{H}), 7.62-7.54$ (m, $4 \mathrm{H}), 7.45-7.44(\mathrm{~m}, 2 \mathrm{H}), 6.92$ (s, $1 \mathrm{H}), 3.67(\mathrm{~s}, 3 \mathrm{H}) .{ }^{13} \mathrm{C}$ NMR $\left(125 \mathrm{MHz}\right.$, DMSO- $\left.d_{6}\right): \delta=189.6$, 156.1, 144.4, 139.0. 132.6, 130.4, 130.2, 129.3, 128.7, 127.9, 127.6, 127.0, 126.1, 125.8, 124.9, 113.1, 112.2, 91.1, 30.4. HRMS (ESI): $m / z[\mathrm{M}+\mathrm{H}]^{+}$calcd for $\mathrm{C}_{21} \mathrm{H}_{16} \mathrm{~N}_{2} \mathrm{O}_{2}$ : 329.1290; found: 329.1285 .

(Z)-6,7-Dibromo-1-methyl-3-(2-oxo-2-phenylethylidene)3,4-dihydroquinoxalin-2(1H)-one (3an)

Yield 51\%; yellow solid. ${ }^{1} \mathrm{H}$ NMR (500 MHz, DMSO- $\left.d_{6}\right): \delta=13.35$ $(\mathrm{s}, 1 \mathrm{H}), 8.01(\mathrm{~s}, 1 \mathrm{H}), 7.92(\mathrm{~d}, J=5 \mathrm{~Hz}, 2 \mathrm{H}), 7.61-7.49(\mathrm{~m}, 4 \mathrm{H})$, $6.81(\mathrm{~s}, 1 \mathrm{H}), 3.50(\mathrm{~s}, 3 \mathrm{H}) .{ }^{13} \mathrm{C}$ NMR $\left(125 \mathrm{MHz}\right.$, DMSO-d $\left.d_{6}\right): \delta=$ 188.9, 155.6, 144.0, 138.7, 132.6, 129.2, 127.5, 125.9, 121.4, 119.6, 118.3, 118.1, 91.3, 30.4. HRMS (ESI): $\mathrm{m} / \mathrm{z}[\mathrm{M}+\mathrm{H}]^{+}$calcd for $\mathrm{C}_{17} \mathrm{H}_{12} \mathrm{Br}_{2} \mathrm{~N}_{2} \mathrm{O}_{2}$ : 434.9344; found: 434.9349 .

(Z)-3-[2-(2-Hydroxyphenyl)-2-oxoethylidene]-1-methyl-3,4dihydroquinoxalin-2(1H)-one (3ba)

Yield 57\%; yellow solid; mp 207-209 ${ }^{\circ} \mathrm{C} .{ }^{1} \mathrm{H}$ NMR $(400 \mathrm{MHz}$, DMSO- $\left.d_{6}\right): \delta=13.27(\mathrm{~s}, 1 \mathrm{H}), 12.85(\mathrm{~s}, 1 \mathrm{H}), 7.90(\mathrm{~d}, J=7.7 \mathrm{~Hz}, 1$ H), $7.68(\mathrm{~d}, J=7.4 \mathrm{~Hz}, 1 \mathrm{H}), 7.48-7.44(\mathrm{~m}, 2 \mathrm{H}), 6.97-6.93(\mathrm{~m}, 2$ H), $3.61(\mathrm{~s}, 3 \mathrm{H}) .{ }^{13} \mathrm{C}$ NMR $\left(101 \mathrm{MHz}\right.$, DMSO- $\left.d_{6}\right): \delta=188.9,167.4$, $156.2,146.1,139.1,132.4,129.2,127.5,127.2,124.6,124.5$, 124.2, 117.1, 115.8, 89.6, 40.0. HRMS (ESI): $m / z[\mathrm{M}+\mathrm{H}]^{+}$calcd for $\mathrm{C}_{17} \mathrm{H}_{14} \mathrm{~N}_{2} \mathrm{O}_{3}$ : 295.1083; found: 295.1079.

(Z)-1-Methyl-3-[2-oxo-2-(o-tolyl)ethylidene]-3,4-dihydroquinoxalin-2(1H)-one (3bb)

Yield 33\%; yellow solid; mp $144-147{ }^{\circ} \mathrm{C} .{ }^{1} \mathrm{H}$ NMR $(500 \mathrm{MHz}$, DMSO- $\left.d_{6}\right): \delta=13.54(\mathrm{~s}, 1 \mathrm{H}), 7.58-7.54(\mathrm{~m}, 2 \mathrm{H}), 7.42-7.37(\mathrm{~m}$, $2 \mathrm{H}), 7.32-7.22(\mathrm{~m}, 4 \mathrm{H}), 6.44(\mathrm{~s}, 1 \mathrm{H}), 3.58(\mathrm{~s}, 3 \mathrm{H}), 2.46(\mathrm{~s}, 3 \mathrm{H})$. ${ }^{13} \mathrm{C}$ NMR $\left(125 \mathrm{MHz}\right.$, DMSO- $\left.d_{6}\right): \delta=193.8,155.7,144.3,140.7$, $136.2,131.7,130.5,128.7,128.1,126.4,125.0,124.6,124.4$ 117.4, 115.5, 93.9, 30.2, 20.5. HRMS (ESI): $m / z[\mathrm{M}+\mathrm{H}]^{+}$calcd for $\mathrm{C}_{18} \mathrm{H}_{16} \mathrm{~N}_{2} \mathrm{O}_{2}$ : 293.1290; found: 293.1295.

(Z)-3-[2-(4-Methoxyphenyl)-2-oxoethylidene]-1-methyl-3,4dihydroquinoxalin-2(1H)-one (3bc)

Yield 74\%; yellow solid; mp $199-203{ }^{\circ} \mathrm{C} .{ }^{1} \mathrm{H}$ NMR (400 MHz, DMSO- $\left.d_{6}\right): \delta=13.76(\mathrm{~s}, 1 \mathrm{H}), 7.98(\mathrm{~d}, J=10.8 \mathrm{~Hz}, 2 \mathrm{H}), 7.53-7.51$ $(\mathrm{m}, 1 \mathrm{H}), 7.42-7.40(\mathrm{~m}, 1 \mathrm{H}), 7.24-7.21(\mathrm{~m}, 2 \mathrm{H}), 7.08(\mathrm{~d}, J=8.8$ $\mathrm{Hz}, 2 \mathrm{H}), 6.83(\mathrm{~s}, 1 \mathrm{H}), 3.85(\mathrm{~s}, 3 \mathrm{H}), 3.60(\mathrm{~s}, 3 \mathrm{H}) .{ }^{13} \mathrm{C}$ NMR (100 MHz, DMSO- $\left.d_{6}\right): \delta=188.1,162.9,156.0,144.6,131.8,129.6$, 128.6, 125.2, 124.5, 124.4, 117.1, 115.5, 114.5, 89.8, 55.9, 30.2. HRMS (ESI): $m / z[M+H]^{+}$calcd for $\mathrm{C}_{18} \mathrm{H}_{16} \mathrm{~N}_{2} \mathrm{O}_{3}$ : 309.1239; found: 309.1238 .

(Z)-1-Methyl-3-[2-oxo-2-(p-tolyl)ethylidene]-3,4-dihydroquinoxalin-2(1H)-one (3bd)

Yield 62\%; yellow solid; mp $179-181{ }^{\circ} \mathrm{C} .{ }^{1} \mathrm{H}$ NMR $(500 \mathrm{MHz}$, DMSO- $\left._{6}\right): \delta=13.79(\mathrm{~s}, 1 \mathrm{H}), 7.88(\mathrm{~d}, J=8.0 \mathrm{~Hz}, 2 \mathrm{H}), 7.53(\mathrm{~d}, J=$ $9.0 \mathrm{~Hz}, 2 \mathrm{H}), 7.41(\mathrm{~d}, J=7.2 \mathrm{~Hz}, 2 \mathrm{H}), 7.33(\mathrm{~d}, J=7.9 \mathrm{~Hz}, 2 \mathrm{H})$, 7.25-7.20 (m, $2 \mathrm{H}), 6.82$ (s, $1 \mathrm{H}), 3.58(\mathrm{~s}, 3 \mathrm{H}), 2.37$ (s, $3 \mathrm{H}) .{ }^{13} \mathrm{C}$ NMR $\left(125 \mathrm{MHz}\right.$, DMSO- $\left.d_{6}\right): \delta=188.5,155.8,144.9,142.6,136.4$, 129.8, 128.7, 127.5, 125.1, 124.5, 117.3, 115.5, 89.9, 30.2, 21.6. HRMS (ESI): $\mathrm{m} / z[\mathrm{M}+\mathrm{H}]^{+}$calcd for $\mathrm{C}_{18} \mathrm{H}_{16} \mathrm{~N}_{2} \mathrm{O}_{2}:$ 293.1290; found: 293.1288 .

(Z)-3-[2-(4-Bromophenyl)-2-oxoethylidene]-1-methyl-3,4dihydroquinoxalin-2(1H)-one (3be)

Yield 61\%; yellow solid; mp 263-265 ${ }^{\circ} \mathrm{C} .{ }^{1} \mathrm{H}$ NMR (400 MHz, 
DMSO- $\left.d_{6}\right): \delta=13.84(\mathrm{~s}, 1 \mathrm{H}), 7.94(\mathrm{~d}, J=8.6 \mathrm{~Hz}, 2 \mathrm{H}), 7.75(\mathrm{~d}, J=$ 8.6 Hz, $2 \mathrm{H}), 7.60-7.58$ (m, $1 \mathrm{H}), 7.45-7.43(\mathrm{~m}, 1 \mathrm{H}), 7.30-7.22$ $(\mathrm{m}, 2 \mathrm{H}), 6.84(\mathrm{~s}, 1 \mathrm{H}), 3.61(\mathrm{~s}, 3 \mathrm{H}) .{ }^{13} \mathrm{C}$ NMR $(100 \mathrm{MHz}$, DMSO$\left.d_{6}\right): \delta=187.4,155.7,145.5,138.1,132.3,129.5,128.9,126.3$, 124.9, 124.6, 117.6, 115.6, 100.0, 89.7, 30.3. HRMS (ESI): $\mathrm{m} / z$ [M $+\mathrm{H}]^{+}$calcd for $\mathrm{C}_{17} \mathrm{H}_{13} \mathrm{BrN}_{2} \mathrm{O}_{2}$ : 357.0239; found: 357.0241 . (Z)-3-[2-(4-Chlorophenyl)-2-oxoethylidene]-1-methyl-3,4dihydroquinoxalin-2(1H)-one (3bf)

Yield 51\%; yellow solid; mp $248-253{ }^{\circ} \mathrm{C} .{ }^{1} \mathrm{H}$ NMR (400 MHz, DMSO- $\left.d_{6}\right): \delta=13.83(\mathrm{~s}, 1 \mathrm{H}), 8.01(\mathrm{~d}, J=8.6 \mathrm{~Hz}, 2 \mathrm{H}), 7.60$ (d, $J=$ $8.6 \mathrm{~Hz}, 2 \mathrm{H}), 7.44(\mathrm{~d}, J=7.7 \mathrm{~Hz}, 2 \mathrm{H}), 7.28-7.24(\mathrm{~m}, 2 \mathrm{H}), 6.84(\mathrm{~s}$, $1 \mathrm{H}), 3.60$ (s, $3 \mathrm{H}) .{ }^{13} \mathrm{C}$ NMR (100 MHz, DMSO- $\left.d_{6}\right): \delta=187.2$, 155.7, 145.4, 137.7, 137.2, 129.4, 128.9, 125.0, 124.6, 117.6, 115.6, 89.7, 30.3. HRMS (ESI): $m / z[M+H]^{+}$calcd for $\mathrm{C}_{17} \mathrm{H}_{13^{-}}$ $\mathrm{ClN}_{2} \mathrm{O}_{2}$ : 313.0744; found: 313.0742 .

\section{(Z)-3-[2-(4-Iodophenyl)-2-oxoethylidene]-1-methyl-3,4-} dihydroquinoxalin-2(1H)-one (3bg)

Yield 49\%; yellow solid; mp 204-206 ${ }^{\circ} \mathrm{C} .{ }^{1} \mathrm{H}$ NMR $(400 \mathrm{MHz}$, DMSO- $\left.d_{6}\right): \delta=13.83(\mathrm{~s}, 1 \mathrm{H}), 7.92(\mathrm{~d}, J=8 \mathrm{~Hz}, 2 \mathrm{H}), 7.76(\mathrm{~d}, J=$ $8.5 \mathrm{~Hz}, 2 \mathrm{H}), 7.58-7.56(\mathrm{~m}, 1 \mathrm{H}), 7.44-7.42(\mathrm{~m}, 1 \mathrm{H}), 7.29-7.21$ $(\mathrm{m}, 2 \mathrm{H}), 6.82(\mathrm{~s}, 1 \mathrm{H}), 3.60(\mathrm{~s}, 3 \mathrm{H}) .{ }^{13} \mathrm{C}$ NMR (100 MHz, DMSO$\left.d_{6}\right): \delta=187.6,155.7,145.4,138.4,138.2,129.3,128.9,125.0$, 124.9, 124.6, 117.6, 115.6, 100.4, 89.6, 30.3. HRMS (ESI): $m / z$ [M $+\mathrm{H}]^{+}$calcd for $\mathrm{C}_{17} \mathrm{H}_{13} \mathrm{IN}_{2} \mathrm{O}_{2}: 405.0100$; found: 405.0102 .

(Z)-3-[2-(2-Fluorophenyl)-2-oxoethylidene]-1-methyl-3,4dihydroquinoxalin-2(1H)-one (3bh)

Yield 70\%; yellow solid; mp $145-150{ }^{\circ} \mathrm{C} .{ }^{1} \mathrm{H}$ NMR $(500 \mathrm{MHz}$, DMSO- $\left.d_{6}\right): \delta=13.75(\mathrm{~s}, 1 \mathrm{H}), 7.88-7.84(\mathrm{~m}, 1 \mathrm{H}), 7.60(\mathrm{~d}, J=6.5$ $\mathrm{Hz}, 2 \mathrm{H}), 7.44$ (d, J = 7.9 Hz, $2 \mathrm{H}), 7.37-7.32(\mathrm{~m}, 2 \mathrm{H}), 7.29-7.22$ (m, $2 \mathrm{H}), 6.75$ (s, $1 \mathrm{H}), 3.59$ (s, $3 \mathrm{H}) .{ }^{13} \mathrm{C}$ NMR $(125 \mathrm{MHz}$, DMSO$\left.d_{6}\right): \delta=185.4,161.5,157.5,155.6,145.2,134.0,133.9(J=35.5$ $\mathrm{Hz}), 130.5,129.0,127.7,127.6(J=49.0 \mathrm{~Hz}), 125.3(J=13.0 \mathrm{~Hz})$, 125.0, 124.9, 124.5, 117.7, $117.2(J=250.6 \mathrm{~Hz}), 117.0,115.6$, 94.2, 30.3. HRMS (ESI): $m / z[\mathrm{M}+\mathrm{H}]^{+}$calcd for $\mathrm{C}_{17} \mathrm{H}_{13} \mathrm{FN}_{2} \mathrm{O}_{2}$ : 297.1039; found: 297.1041 .

(Z)-1-Methyl-3-\{2-oxo-2-[2-(trifluoromethyl)phenyl]ethylidene\}-3,4-dihydroquinoxalin-2(1H)-one (3bi) Yield 35\%; yellow solid; mp 191-195 ${ }^{\circ} \mathrm{C} .{ }^{1} \mathrm{H}$ NMR $(500 \mathrm{MHz}$, DMSO- $\left.d_{6}\right): \delta=13.29(\mathrm{~s}, 1 \mathrm{H}), 7.84-7.76(\mathrm{~m}, 2 \mathrm{H}), 7.65-7.70(\mathrm{~m}, 3$ H), $7.43(\mathrm{~d}, J=7.7 \mathrm{~Hz}, 1 \mathrm{H}), 7.28-7.21,(\mathrm{~m}, 2 \mathrm{H}), 6.32(\mathrm{~s}, 1 \mathrm{H})$, $3.58(\mathrm{~s}, 3 \mathrm{H}) .{ }^{13} \mathrm{C}$ NMR $\left(125 \mathrm{MHz}\right.$, DMSO- $\left.d_{6}\right): \delta=191.7,155.4$, 144.6, 140.9, 133.1, 130.6, 128.9, $128.8(J=65.4 \mathrm{~Hz}), 127.1$, $127.0(J=21.2 \mathrm{~Hz}), 126.2,126.0(J=124.8 \mathrm{~Hz}), 125.5,125.0$, 124.7, $124.5(\mathrm{~J}=61.7 \mathrm{~Hz}), 123.3,117.6,115.6,93.5$, 30.3. HRMS (ESI): $m / z[M+H]^{+}$calcd for $\mathrm{C}_{18} \mathrm{H}_{13} \mathrm{~F}_{3} \mathrm{~N}_{2} \mathrm{O}_{2}$ : 347.1007; found: 347.1002.

(Z)-1-Methyl-3-[2-(naphthalen-2-yl)-2-oxoethylidene]-3,4dihydroquinoxalin-2(1H)-one (3bj)

Yield 78\%; yellow solid; mp $164-167{ }^{\circ} \mathrm{C} .{ }^{1} \mathrm{H}$ NMR $(400 \mathrm{MHz}$, DMSO- $\left.d_{6}\right): \delta=13.69(\mathrm{~s}, 1 \mathrm{H}), 8.52(\mathrm{~d}, J=7.6 \mathrm{~Hz}, 1 \mathrm{H}), 8.09(\mathrm{~d}, J=$ $6.6 \mathrm{~Hz}, 1 \mathrm{H}), 8.02-8.01(\mathrm{~m}, 1 \mathrm{H}), 7.84(\mathrm{~d}, J=5.6 \mathrm{~Hz}, 1 \mathrm{H}), 7.62-$ $7.59(\mathrm{~m}, 4 \mathrm{H}), 7.43(\mathrm{~d}, J=6.0 \mathrm{~Hz}, 1 \mathrm{H}), 7.29-7.23(\mathrm{~m}, 2 \mathrm{H}), 6.61(\mathrm{~s}$, $1 \mathrm{H}), 3.59(\mathrm{~s}, 3 \mathrm{H}) .{ }^{13} \mathrm{C}$ NMR (101 MHz, DMSO- $\left.d_{6}\right): \delta=193.2$, $155.8,144.6,138.6,133.9,131.4,130.0,128.9,128.8,127.5$, 126.8, 126.7, 126.0, 125.7, 125.0, 124.7, 124.5, 117.4, 115.6, 94.5, 30.2. HRMS (ESI): $m / z[M+H]^{+}$calcd for $\mathrm{C}_{21} \mathrm{H}_{16} \mathrm{~N}_{2} \mathrm{O}_{2}$ : 329.1290; found: 329.1286 .

1-Methyl-3-phenylquinoxalin-2(1H)-one (4a)

Yield 88\%; white solid; mp $114-116{ }^{\circ} \mathrm{C} .{ }^{1} \mathrm{H}$ NMR $(500 \mathrm{MHz}$, $\left.\mathrm{CDCl}_{3}\right): \delta=8.31-8.30(\mathrm{~m}, 2 \mathrm{H}), 7.95(\mathrm{~d}, J=9.2 \mathrm{~Hz}, 1 \mathrm{H}), 7.59(\mathrm{t}, J=$ 7.1 Hz, $1 \mathrm{H}), 7.49-7.48$ (m, $3 \mathrm{H}), 7.39-7.34$ ( $\mathrm{m}, 2 \mathrm{H}), 3.78$ (s, $3 \mathrm{H})$. ${ }^{13} \mathrm{C}$ NMR $\left(125 \mathrm{MHz}, \mathrm{CDCl}_{3}\right): \delta=154.8,154.2,136.1,133.4,133.1$, 130.5, 130.3, 129.6, 128.1, 123.8, 113.6, 29.3. HRMS (ESI): $\mathrm{m} / \mathrm{z}$
$[\mathrm{M}+\mathrm{H}]^{+}$calcd for $\mathrm{C}_{15} \mathrm{H}_{12} \mathrm{~N}_{2} \mathrm{O}$ : 237.1028; found: 237.1028 . Ethyl 2-[2-0xo-3-phenylquinoxalin-1(2H)-yl]acetate (4aa) Yield 47\%; white solid. ${ }^{1} \mathrm{H}$ NMR $\left(400 \mathrm{MHz}, \mathrm{CDCl}_{3}\right): \delta=8.33-8.31$ (m, $2 \mathrm{H}), 7.99$ (dd, $\left.J_{1}=8.0 \mathrm{~Hz}, J_{2}=1.4 \mathrm{~Hz}, 1 \mathrm{H}\right), 7.57-7.52(\mathrm{~m}, 1$ H), 7.51-7.47 (m, $3 \mathrm{H}), 7.41-7.36(\mathrm{~m}, 1 \mathrm{H}), 7.13(\mathrm{~d}, J=8.4 \mathrm{~Hz}, 1$ $\mathrm{H}), 5.10(\mathrm{~s}, 2 \mathrm{H}), 4.30(\mathrm{q}, J=7.2 \mathrm{~Hz}, 2 \mathrm{H}), 1.31(\mathrm{t}, J=7.1 \mathrm{~Hz}, 3 \mathrm{H})$. ${ }^{13} \mathrm{C} \mathrm{NMR}\left(100 \mathrm{MHz}, \mathrm{CDCl}_{3}\right): \delta=167.2,154.3,154.0,135.7,133.2$, 132.5, 130.8, 130.5, 129.6, 128.1, 124.1, 113.1, 62.1, 43.8, 14.2. HRMS (ESI): $m / z[M+H]^{+}$calcd for $\mathrm{C}_{18} \mathrm{H}_{16} \mathrm{~N}_{2} \mathrm{O}_{3}: 309.1239$; found: 309.1238 .

\section{1-[4-(tert-Butyl)benzyl]-3-phenylquinoxalin-2(1H)-one} (4ab)

Yield 37\%; white solid. ${ }^{1} \mathrm{H}$ NMR ( $\left.400 \mathrm{MHz}, \mathrm{CDCl}_{3}\right): \delta=8.29-8.27$ (m, $2 \mathrm{H}), 7.89$ (d, $J=6.4 \mathrm{~Hz}, 1 \mathrm{H}), 7.43-7.41(\mathrm{~m}, 4 \mathrm{H}), 7.28-7.25$ (m, $5 \mathrm{H}), 7.18$ (s, $2 \mathrm{H}), 5.47$ (s, $2 \mathrm{H}), 1.20(\mathrm{~s}, 9 \mathrm{H}) .{ }^{13} \mathrm{C}$ NMR (100 $\left.\mathrm{MHz}, \mathrm{CDCl}_{3}\right): \delta=154.8,154.3,150.7,136.1,133.4,132.9,132.3$, 130.6, 130.4, 130.3, 129.7, 128.1, 126.8, 125.9, 123.8, 114.5, 45.9, 34.5, 31.3. HRMS (ESI): $m / z[\mathrm{M}+\mathrm{H}]^{+}$calcd for $\mathrm{C}_{25} \mathrm{H}_{24} \mathrm{~N}_{2} \mathrm{O}$ : 369.1967; found: 369.1669 .

1-Benzyl-3-phenylquinoxalin-2(1H)-one (4ac)

Yield 51\%; white solid. ${ }^{1} \mathrm{H}$ NMR $\left(400 \mathrm{MHz}, \mathrm{CDCl}_{3}\right): \delta=8.38-8.35$ (m, $2 \mathrm{H}), 7.97$ (d, $J=9.4 \mathrm{~Hz}, 1 \mathrm{H}), 7.51-7.49(\mathrm{~m}, 3 \mathrm{H}), 7.46-7.43$ $(\mathrm{m}, 1 \mathrm{H}), 7.36-7.26(\mathrm{~m}, 7 \mathrm{H}), 5.58(\mathrm{~s}, 2 \mathrm{H}) .{ }^{13} \mathrm{C}$ NMR $(100 \mathrm{MHz}$, $\left.\mathrm{CDCl}_{3}\right): \delta=154.8,154.3,136.0,135.4,133.4,132.8,130.6,130.5$, 130.4, 129.7, 129.0, 128.1, 127.7, 127.0, 123.8, 114.4, 46.2. HRMS (ESI): $m / z[\mathrm{M}+\mathrm{H}]^{+}$calcd for $\mathrm{C}_{21} \mathrm{H}_{16} \mathrm{~N}_{2} \mathrm{O}$ : 313.1341 ; found: 313.1343.

1-(2-0xo-2-phenylethyl)-3-phenylquinoxalin-2(1H)-one (4ad)

Yield 32\%; white solid. ${ }^{1} \mathrm{H}$ NMR ( $400 \mathrm{MHz}, \mathrm{CDCl}_{3}$ ): $\delta=8.34-8.31$ $(\mathrm{m}, 2 \mathrm{H}), 8.12-8.10(\mathrm{~m}, 2 \mathrm{H}), 8.01\left(\mathrm{dd}, J_{1}=8.0 \mathrm{~Hz}, J_{2}=1.4 \mathrm{~Hz}, 1\right.$ H), 7.71-7.67 (m, $2 \mathrm{H}), 7.59-7.55(\mathrm{~m}, 2 \mathrm{H}), 7.49-7.48(\mathrm{~m}, 4 \mathrm{H})$, 7.39-7.35 (m, $1 \mathrm{H}), 7.01(\mathrm{~d}, J=9.1 \mathrm{~Hz}, 1 \mathrm{H}), 5.82(\mathrm{~s}, 2 \mathrm{H}) .{ }^{13} \mathrm{C}$ NMR $\left(101 \mathrm{MHz}\right.$, DMSO- $\left.d_{6}\right): \delta=192.9,154.3,153.2,136.0,135.0$, 133.4, 132.8, 131.2, 130.9, 130.3, 129.8, 129.5, 129.0, 128.8, $128.5,124.3,115.9,50.9$. HRMS (ESI): $m / z[\mathrm{M}+\mathrm{H}]^{+}$calcd for $\mathrm{C}_{22} \mathrm{H}_{16} \mathrm{~N}_{2} \mathrm{O}_{2}$ : 341.1290; found: 341.1290 .

\section{3-Phenyl-1-propylquinoxalin-2(1H)-one (4ae)}

Yield $28 \%$; colorless oily liquid. ${ }^{1} \mathrm{H}$ NMR $\left(400 \mathrm{MHz}, \mathrm{CDCl}_{3}\right): \delta=$ 8.32-8.30 (m, $2 \mathrm{H}), 7.97-7.95(\mathrm{~m}, 1 \mathrm{H}), 7.58-7.54(\mathrm{~m}, 1 \mathrm{H})$, 7.49-7.47 (m, $3 \mathrm{H}), 7.38-7.33(\mathrm{~m}, 2 \mathrm{H}), 4.31-4.27(\mathrm{~m}, 2 \mathrm{H})$, $1.90-1.81(\mathrm{~m}, 2 \mathrm{H}), 1.11(\mathrm{t}, J=7.4 \mathrm{~Hz}, 3 \mathrm{H}) .{ }^{13} \mathrm{C}$ NMR $(100 \mathrm{MHz}$, $\left.\mathrm{CDCl}_{3}\right): \delta=154.4,154.2,136.1,133.4,132.6,130.7,130.3,130.2$, 129.6, 128.1, 123.5, 113.6, 44.1, 20.7, 11.5. HRMS (ESI): $\mathrm{m} / z$ [M + $\mathrm{H}]^{+}$calcd for $\mathrm{C}_{17} \mathrm{H}_{17} \mathrm{~N}_{2} \mathrm{O}$ : 265.1341; found: 265.1345.

1,3-Diphenylquinoxalin-2(1H)-one (4af)

Yield 94\%; white solid. ${ }^{1} \mathrm{H}$ NMR $\left(400 \mathrm{MHz}, \mathrm{CDCl}_{3}\right): \delta=8.40-8.38$ ( $\mathrm{m}, 2 \mathrm{H}$ ), 8.00-7.98 (m, $2 \mathrm{H}$ ), 7.66-7.56 ( $\mathrm{m}, 4 \mathrm{H}), 7.49-7.47$ ( $\mathrm{m}, 3$ $\mathrm{H}), 7.37-7.34(\mathrm{~m}, 4 \mathrm{H}) .{ }^{13} \mathrm{C}$ NMR $\left(100 \mathrm{MHz}, \mathrm{CDCl}_{3}\right): \delta=154.5$, $136.1,135.7,134.2,133.0,130.5,130.3,130.1,130.0,129.7$, 129.4, 128.3, 128.1, 123.9, 115.4. HRMS (ESI): $m / z[\mathrm{M}+\mathrm{H}]^{+}$calcd for $\mathrm{C}_{20} \mathrm{H}_{14} \mathrm{~N}_{2} \mathrm{O}$ : 299.1184; found: 299.1180.

1,6,7-Trimethyl-3-phenylquinoxalin-2(1H)-one (4ag) Yield 66\%; white solid; mp $154-156{ }^{\circ} \mathrm{C} .{ }^{1} \mathrm{H}$ NMR $(400 \mathrm{MHz}$, $\left.\mathrm{CDCl}_{3}\right): \delta=8.29-8.26(\mathrm{~m}, 2 \mathrm{H}), 7.71(\mathrm{~s}, 1 \mathrm{H}), 8.49-8.35(\mathrm{~m}, 4 \mathrm{H})$, 7.11 (s, $1 \mathrm{H}), 3.76(\mathrm{~s}, 3 \mathrm{H}), 2.45$ (s, $3 \mathrm{H}), 2.38$ (s, $3 \mathrm{H}) .{ }^{13} \mathrm{C}$ NMR $\left(101 \mathrm{MHz}\right.$, DMSO- $\left.d_{6}\right): \delta=167.8,154.5,152.1,140.9,137.0$, 133.4, 132.8, 131.2, 129.7, 129.1, 128.3, 115.5, 29.7, 20.5, 19.2. HRMS (ESI): $m / z$ [M $+\mathrm{H}]^{+}$calcd for $\mathrm{C}_{17} \mathrm{H}_{16} \mathrm{~N}_{2} \mathrm{O}: 265.1341$; found: 265.1338 .

6,7-Chloro-1-methyl-3-phenylquinoxalin-2(1H)-one (4ah) Yield 95\%; white solid; mp $172-174{ }^{\circ} \mathrm{C} .{ }^{1} \mathrm{H}$ NMR $(400 \mathrm{MHz}$, $\left.\mathrm{CDCl}_{3}\right): \delta=8.31-8.29(\mathrm{~m}, 2 \mathrm{H}), 8.02(\mathrm{~s}, 1 \mathrm{H}), 7.51-7.47(\mathrm{~m}, 3 \mathrm{H})$, 
$7.42(\mathrm{~s}, 1 \mathrm{H}), 3.72(\mathrm{~s}, 3 \mathrm{H}) .{ }^{13} \mathrm{C}$ NMR $\left(100 \mathrm{MHz}, \mathrm{CDCl}_{3}\right): \delta=155.2$, 154.1, 135.4, 134.3, 132.7, 132.2, 131.1, 130.9, 130.0, 128.2, 115.1, 29.6. HRMS (ESI): $\mathrm{m} / z$ [M $+\mathrm{H}]^{+}$calcd for $\mathrm{C}_{15} \mathrm{H}_{10} \mathrm{Cl}_{2} \mathrm{~N}_{2} \mathrm{O}$ : 306.1660; found: 306.1661.

1-Methyl-3-phenylbenzo[g]quinoxalin-2(1H)-one (4ai)

Yield 70\%; yellow solid; mp $162-164{ }^{\circ} \mathrm{C} .{ }^{1} \mathrm{H}$ NMR (400 MHz, $\left.\mathrm{CDCl}_{3}\right): \delta=8.45(\mathrm{~s}, 1 \mathrm{H}), 8.34-8.32(\mathrm{~m}, 2 \mathrm{H}), 8.00-7.91(\mathrm{~m}, 2 \mathrm{H})$, $7.62(\mathrm{~s}, 1 \mathrm{H}), 7.59-7.55(\mathrm{~m}, 1 \mathrm{H}), 7.51-7.49(\mathrm{~m}, 4 \mathrm{H}), 3.83(\mathrm{~s}, 3$ H). ${ }^{13} \mathrm{C}$ NMR $\left(100 \mathrm{MHz}, \mathrm{CDCl}_{3}\right): \delta=154.8,154.7,136.1,133.8$, 132.5, 132.0, 130.5, 129.9, 129.7, 129.7, 128.5, 128.1, 127.9, 127.2, 125.3, 109.8, 29.3. HRMS (ESI): $m / z[M+H]^{+}$calcd for $\mathrm{C}_{19} \mathrm{H}_{14} \mathrm{~N}_{2} \mathrm{O}$ : 287.3420; found: 287.3421 .

(28) Carrër, A.; Brion, J.-D.; Messaoudi, S.; Alami, M. Org. Lett. 2013, $15,5606$. 